\title{
Importancia nutricional y actividad biológica de los compuestos bioactivos de quelites consumidos en México
}

\section{Nutritional importance and biological activity of bioactive compounds from quelites consumed in Mexico}

\begin{abstract}
RESUMEN
Sólo un 30\% de los hogares en México, presentan seguridad alimentaria, es decir, un gran porcentaje de los hogares y por ende de la población no satisfacen las necesidades básicas diarias de macro y micronutrimentos. Los quelites, que se definen como plantas silvestres comestibles (hojas, tallos y flores) son una fuente accesible, continua, económica y adecuada de nutrimentos. Sin embargo, su consumo ha disminuido por modificaciones en las preferencias alimentarias, derivadas de los cambios en estilos de vida. El objetivo del presente trabajo fue valorizar a los quelites como fuente de alimento. Los quelites, aportan proteínas, aminoácidos, minerales ( $C a, M g, Z n)$, vitaminas $(E, C)$ y fibra. Además, son una excelente fuente de compuestos bioactivos, como ácidos fenólicos (ácido cafeico, ferúlico) y flavonoides (quercetina, kaempferol, espinacetina), carotenoides, ácido $\alpha$-linolénico y betalainas, que presentan elevada actividad antioxidante. Su consumo habitual se ha relacionado con beneficios a la salud, tales como efectos antitumorales, antihiperlipidémicos y antidiabéticos. Los quelites, además de estar disponibles en forma silvestre, forman parte de las tradiciones culinarias de México, incorporados de forma cruda y cocida en los platillos regionales. Por lo tanto, la revalorización y reincorporación de los quelites en la dieta, puede coadyuvar a cubrir las necesidades nutrimentales, en poblaciones con poco acceso o inseguridad alimentaria, además de contribuir a proporcionar efectos adicionales a través de sus compuestos bioactivos.

Palabras clave: Alimento tradicional; Enfermedades crónicas no transmisibles; Plantas silvestres; Quelites; Seguridad alimentaria.
\end{abstract}

\footnotetext{
ABSTRACT

Only 30\% of households in Mexico present food security, which means a large percentage of households and the population does not meet their needs in terms of macro and micronutrients. Thus, quelites, which are defined as wild edible plants, are an accessible, continuous, economical and adequate source of nutrients. However, quelite consumption in Mexico has been decreasing in response to changes in food preferences and lifestyles, including
}

Yair O Santiago-Saenz ${ }^{1}$, Alma D Hernández-Fuentes ${ }^{1}$, César U López-Palestina², Jesús H Garrido-Cauich ${ }^{3}$, Julia Ma Alatorre-Cruz ${ }^{4}$, Rebeca Monroy-Torres ${ }^{5 *}$.

\begin{abstract}
1. Área Académica de Agroindustrial. Instituto de Ciencias Agropecuarias. Universidad Autónoma del Estado de Hidalgo. Rancho Universitario. Hidalgo, México.

2. División de Posgrado. Tecnológico Nacional de México-Instituto Tecnológico de Roque, Guanajuato, México. 3. Departamento de Nutrición. Servicios de Salud de Yucatán. Secretaría de Salud. Jurisdicción sanitaria Núm. 3 Ticul. Yucatán, México. 4. Departamento de Biología. Facultad de Ciencias Naturales. Universidad Autónoma de Querétaro. Santiago de Querétaro, Querétaro, México.

5. Laboratorio de Nutrición Ambiental y Seguridad Alimentaria. Departamento de Medicina y Nutrición, División de Ciencias de la Salud, Universidad de Guanajuato. León, Gto.
\end{abstract}

\begin{abstract}
* Dirigir correspondencia: Rebeca Monroy Torres. Laboratorio de Nutrición Ambiental y Seguridad Alimentaria. Departamento de Medicina y Nutrición, Campus León, Universidad de Guanajuato. Tel: (+52) 4772674900, Ext 3677. E-mail: rmonroy79@gmail.com
\end{abstract}

Este trabajo fue recibido el 07 de julio de 2018. Aceptado con modificaciones: 03 de enero de 2019 . Aceptado para ser publicado: 15 de abril de 2019 .

increased exposure to hypercaloric foods. Therefore, the aim of this work was to discuss the value of quelites as a food source rich in nutrients, proteins, amino acids, minerals $(C a, M g, Z n)$, vitamins $(E, C)$ and fiber. We also discuss how quelites are good source of bioactive compounds, such as phenolic acids (caffeic acid, ferulic acid) and flavonoids (quercetin, kaempferol, spinacetin), carotenoids, $\alpha$-linolenic acid and betalaines. There use have been mainly related to the anti-tumor, antihyperlipidemic and antidiabetic benefits. We further discuss topics related to the culinary traditions of Mexico and the incorporation of quelites in the raw and cooked form in regional markets. The revaluation and the reincorporation of quelites in the diet can help meet nutritional needs, in addition to possibly providing additional 
health benefits.

Keywords: Cardiometabolic diseases; Food security; Quelites; Traditional food; Wild plants.

\section{INTRODUCCIÓN}

México se ha caracterizado por ser uno de los países al frente en la producción y exportación agroalimentaria siendo el lugar $12^{\circ}$ en lo que respecta en la producción mundial de alimentos y $11^{\circ}$ en producción mundial de cultivos agrícolas, haciendo notable la presencia de alimentos mexicanos en varios continentes alrededor del mundo'. Sin embargo, a pesar de la basta riqueza con la que se cuenta en el país en producción de alimentos, las poblaciones con menor poder adquisitivo y presentes en zonas rurales resultan ser los grupos más desfavorecidos en el acceso de estos, presentando claramente una inseguridad alimentaria $(I A)^{2}$; sin embargo, en México se cuenta con una gran variedad de especies vegetales nativas que pueden contribuir a la nutrición en poblaciones de escasos recursos; entre esas especies se encuentran los quelites ${ }^{3,4}$.

Los quelites son plantas nativas silvestres de México que se han utilizado como alimento desde tiempos prehispánicos y que forman parte de la dieta complementaria del mexicano, aunque en muchas ocasiones llegan a constituir el componente principal de la comida tradicional ${ }^{3,4,5}$. En México se consumen más de 500 especies de quelites distribuidas en diferentes familias botánicas ${ }^{4,6}$, dentro de las cuales destacan especies del género Amaranthus spp. (quintonil), Chenopodium spp. (quelite cenizo) y la especie de Portulaca oleracea (verdolaga), principalmente por considerarse quelites de mayor consumo en la gastronomía mexicana al incorporarse en tamales, sopas, quesadillas o como ensalada ${ }^{6,7}$. Los quelites mexicanos aportan a la dieta diferentes aromas, colores y sabores ${ }^{4}$, además de su contribución en una amplia variedad de nutrimentos como proteína, fibra dietética, nutrimentos inorgánicos $(\mathrm{Ca}, \mathrm{K}, \mathrm{Mg}$, $\mathrm{Mn}, \mathrm{Zn}, \mathrm{P})$, vitaminas $\mathrm{A}$ y $\mathrm{C}^{7,8,9}$ y compuestos bioactivos, tales como compuestos fenólicos, betalaínas, entre otros, los cuales le confieren a estas plantas una elevada capacidad antioxidante ${ }^{9,10}$.

Por otra parte, los quelites se venden de manera común en los mercados locales de los diferentes estados de la República Mexicana, por lo que son accesibles y a bajo costo 7 . Sin embargo, a pesar de la importancia que tienen los quelites como fuente de alimento, así como a su relevancia cultural, culinaria y nutrimental, su uso ha disminuido considerablemente debido a la eliminación química de estos vegetales silvestres, cambio de hábitats de crecimiento ${ }^{7}$ y al cambio en las preferencias de consumo en la dieta mexicana, que ha resultado en la ingesta de alimentos con elevado aporte calórico y en una mayor incidencia en trastornos y enfermedades crónicas no transmisibles, como enfermedades cardiovasculares y diabetes mellitus tipo $2^{2,11}$. Por lo anterior, el propósito de este trabajo es describir la importancia de los quelites de las familias Amaranthaceae y Portulacaceae en la alimentación mexicana y sus efectos potencialmente benéficos a la salud humana por el efecto de los nutrimentos y compuestos bioactivos que contienen.

\section{Generalidades de los quelites}

En México el nombre de quelite se deriva del término náhuatl quilitl el cual ha sido interpretado como hierba comestible. En náhuatl se utilizan algunos términos relacionados como quiltic, verdura verde, o quilyollotli para referirse a los tallos tiernos, retoños o brotes de una planta ${ }^{5,6}$. De acuerdo a la literatura, los quelites pueden ser denominados en diversas lenguas indígenas como kaka (totonaco), Yiwa o yube (mixteco), Xakua (purépecha), Guilibá (Rarámuri), Bok itah (Tzeltal), Itaj (Tzotzil) y K'ani (Ñahñu) ${ }^{5,6}$; por lo cual, los quelites se han definido como plantas que crecen de manera silvestre, de las cuales se consumen las hojas, tallos y flores ${ }^{6,12}$.

El uso de los quelites se remonta a las épocas prehispánicas donde alcanzaron una importancia que figuraba en la clasificación indígena del mundo vivo; sin embargo, el conocimiento y consumo de quelites ha disminuido desde la conquista, ya que los conquistadores no aceptaban las plantas no cultivadas, reemplazando plantas nativas por hierbas introducidas y llevando a una merma en su consumo $^{12}$. En México se consumen aproximadamente 500 especies de quelites ${ }^{4,6}$, las cuales han sido seleccionadas por las tradiciones locales de los distintos pueblos y regiones de los 32 estados de la República Mexicana ${ }^{6}$. Dentro de una clasificación más estricta, en las que se consideran únicamente las hojas tiernas comestibles, se utilizan 358 especies, todas restringidas a las angiospermas y distribuidas en 25 superórdenes, 60 órdenes y 176 géneros $^{12}$. Sin embargo, los quelites mexicanos identificados y analizados hasta el momento ascienden a un aproximado de 250 especies que pertenecen a diferentes familias botánicas ${ }^{4}$. Entre los quelites más comunes se encuentran el pápalo-quelite (Porophylum ruderale subsp. macrocephalum), la verdolaga u oreja de ratón (Portulaca oleracea), el quintonil blanco o rojo (Amaranthus spp.), el romerito (Suaeda nigra), el quelite cenizo, quelite blanco, puerquero o de burro (Chenopodium berlandieri subsp. berlandieri), el huazontle (Chenopodium berlandieri subsp. nuttaliiae), los alaches (Anoda cristata), el epazote (Chenopodium ambrosioides), la chaya de monte o quelite peludo (Cnidoscolus aconitifolius), la hoja santa (Piper auritum) y los chepiles o chipil (Crotalaria spp.) ${ }^{6,13}$. Estas plantas mexicanas presentan en común algunas características taxonómicas, como el subreino (Traqueobionta), superdivisión (Spermatophyta), división (Magnoliophyta) y clase (Magnoliopsida), pero las subclases y ordenes pueden variar (Pápalo-quelite: subclase: Asteridae; orden: Asterales; romerito, huazontle, epazote, quintonil, quelite cenizo, verdolaga: subclase: Caryophyllidae; orden: Caryophyllales; alaches: subclase: Dillenidae; orden: Malvales; chaya: subclase: Rosidae; orden: Euphorbiales; hoja santa: subclase: Magnoliidae; orden: Piperales; chipil: subclase: Rosidae; orden: Fabales) ${ }^{14}$.

Los quelites se encuentran distribuidos en todo el país (desde el sur, centro y norte de México), y es en las 
zonas rurales donde se encuentra la mayor biodiversidad asociada a cultivos como el maíz, fríjol y calabaza ${ }^{15}$. Son usados principalmente para consumo por numerosos grupos étnicos provenientes de Chihuahua, la Huasteca Potosina, Sierra Juárez de Oaxaca, Sierra Norte de Puebla, Chiapas, Tabasco, entre otras comunidades de la República Mexicana? En estas localidades las estructuras vegetales incorporadas a los platillos comprenden las hojas y tallos, los cuales son consumidos en forma cruda, cocida o ligeramente frita, y se combinan con tacos, quesadillas, o sopas. También pueden ser incluidos como condimentos o con propósitos medicinales ${ }^{5,7,13}$; sin embargo, hay que señalar que el estadío de desarrollo de los quelites es importante en la forma de preparación. En las zonas rurales principalmente, los quelites frescos se consumen al inicio del ciclo agrícola, es decir, cuando termina la época de sequía y comienzan las lluvias ${ }^{4,12}$. Muchas de las hierbas que retoñan con la nueva humedad del suelo son comestibles; por lo tanto, las hojas, o plantas enteras consideradas como quelites representan fuentes ricas en proteína, $\mathrm{Ca}, \mathrm{Mg}, \mathrm{Mn}, \mathrm{Zn}, \mathrm{P}$, vitamina $\mathrm{A}$, clorofila y carotenoides por decir algunos ${ }^{9,16}$, los cuales son sumados a la dieta para la subsistencia del campesino y su familia hasta que los cultivos principales sean cosechados ${ }^{17}$.

El quintonil (Amaranthus spp.), el quelite cenizo (Chenopodium berlandieri L.) y la verdolaga (Portulaca oleracea L.) son tres de las plantas más ampliamente distribuidas y consumidas entre la población mexicana, esto se debe a un mayor volumen del producto (consideradas como malezas) obtenido; por lo tanto, se ofertan en un gran número de mercados locales, y su inclusión como ingrediente es considerado en una amplia variedad de platillos (consumo como verdura) ${ }^{7,15}$.

\section{Familia Amaranthaceae, género Amaranthus spp}

Entre las especies de plantas mexicanas pertenecientes al género Amaranthus spp. se encuentran Amaranthus cruentus L., Amaranthus hypochondriacus L. y Amaranthus hybridus L. ${ }^{9,8,18}$. Estos quelites son considerados como las malezas más comunes en México. En algunos estados del país son conocidos como bledo, chichimeca, chongo, lepo, mercolina, ses o quintonil ${ }^{14,18}$. Estos quelites son utilizados en la cocina rural y tradicional para realizar platillos diversos, tales como sopas con limón y tortilla, ensaladas o fritos con un guiso de preferencia ${ }^{7,18}$. El quintonil es considerado como flora nativa mexicana, pero se pueden encontrar otras especies pertenecientes a este género distribuidas en otros países $^{14,18,19}$. Su distribución es mayormente en regiones templadas y tropicales, y su presencia en México se ha registrado en 25 estados $^{14}$. El desarrollo del quintonil, se puede observar como maleza en cultivos de maíz, cafetales, huertos familiares y de traspatio, están fuertemente asociados a las labores agrícolas de los campesinos ${ }^{7}$. El desarrollo de la planta puede suceder en cualquier área que proporcione los nutrientes mínimos, ya que, como todos los quelites, su desarrollo se puede presentar en suelos pobres en nutrientes y agua, lo que los hace tolerantes a la sequía, con bajo mantenimiento (riego) y sin insumos externos (fertilizantes) ${ }^{4,6}$.

Los quintoniles se destacan por ser una planta monoica, anual, erguida y pubescente; de un tamaño de $1 \mathrm{~m}$ o menos y un tallo con rayas longitudinales, a veces rojizo, con frecuencia ramificado; sus hojas presentan láminas foliares ampliamente lanceoladas a ovadas de 3 a $15 \mathrm{~cm}$ de largo por $1 \mathrm{a} 7 \mathrm{~cm}$ de ancho, bases a veces teñidas de rojo y de numerosas flores dispuestas en verticilos muy cercanos entre sí1 ${ }^{14}$.

\section{Familia Amaranthaceae, género Chenopodium spp.}

Las diversas especies del género Chenopodium spp, como Chenopodium berlandieri L., Chenopodium ambrosioides L. y Chenopodium graveolens Willd., son otras de las malezas representativas del territorio mexicano ${ }^{8,9,18}$. Estos quelites pueden encontrarse con nombres populares de quelite cenizo, bledo, choal, quelite de ceniza (Chenopodium berlandieri L.), epazote blanco (Chenopodium ambrosioides L.) y rojo (Chenopodium graveolens Willd.); los cuales son incorporados en la dieta de forma hervida o frita para el caso del quelite cenizo, y como condimento de guisos en caso del epazote, por considerarse una hierba de olor fuerte $^{7,18}$. Chenopodium spp. es considerada una planta con orígenes en América, siendo las especies mencionadas anteriormente como nativas de México ${ }^{8,14,18}$. Su distribución se extiende desde el sur de Canadá hasta Guatemala, y en México se ha localizado en 9 estados $^{14}$; donde crecen espontáneamente en los campos de cultivo y en las orillas de los caminos, por lo que la zona para su desarrollo no depende de un tipo de suelo o condiciones nutrimentales especificas $^{6,7}$. Este tipo de quelites suelen presentarse como una hierba erecta de aproximadamente $2 \mathrm{~m}$ de largo, con tallo simple o ramificado hacia el ápice, anguloso y con rayas longitudinales, de color verde claro o amarillento; sus hojas presentan láminas foliares inferiores oblongas a rómbico-ovadas, las superiores tendiendo a lanceoladas, de $1 \mathrm{a} 13.5 \mathrm{~cm}$ de largo por 0.5 a $8.5 \mathrm{~cm}$ de ancho, de color verde amarillento y de textura harinosa sobre todo en el envés; presenta numerosas flores agrupadas en glomérulos compactos y dispuestos en espigas paniculadas ${ }^{14}$.

\section{Familia Portulacaceae, género Portulaca spp.}

La verdolaga es otra planta silvestre considerada como quelite en México, maleza para los agricultores, pero a la vez alimento popular; la especie es identificada como Portulaca oleracea L. ${ }^{14,18}$. En la cultura mexicana, esta planta es conocida como verdolaga, pitule, uadela, aurrara, xucul, graviol, matacani, mixquilit, chamoico y quelite, ${ }^{74,18}$. Es uno de los quelites más demandados y consumidos en México, por lo que la variedad de guisos puede ser extensa, desde su combinación con carnes rojas, hasta su consumo crudo o en forma hervida acompañada con limón, chile y tortillas de maíz ${ }^{7,18}$. En cuanto a sus orígenes se consideraba durante mucho tiempo que la especie provenía de Europa, sin embargo, otros datos sugieren que se considera como 
una especie nativa de México, aunque también es de origen incierto ${ }^{7,14,18}$. En cuanto a su distribución en el territorio mexicano, se puede encontrar en 22 estados $^{14}$; su desarrollo es favorable en regiones templadas y tropicales como maleza a la orilla de los caminos, ríos y canales, y dentro de parcelas de cultivo y huertos familiares ${ }^{7}$. En cuanto al crecimiento de la verdolaga, adicionalmente a las características que presentan todos los quelites en su desarrollo, esta planta puede propagarse en suelos húmedos, secos, arenosos y arcillosos y presentarse como una de las malezas más agresivas en diversos cultivos como ajo, arroz, avena, fríjol, chile, calabaza, tomate, chícharo entre otros $^{14}$. La verdolaga se destaca por ser una hierba carnosa, rastrera, a veces algo ascendente, alcanzando un tamaño de 5 a $40 \mathrm{~cm}$ de largo; sus tallos en algunas ocasiones son rojizos, ramificados, con las ramas extendidas; sus hojas son alternas, obovado-cuneadas a espatuladas, de 0.5 a 3 cm de largo, por 0.2 a $1.5 \mathrm{~cm}$ de ancho; presentan flores sésiles, solitarias o agrupadas por pocas, rodeadas por escasos pelos inconspicuos ${ }^{14}$.

\section{Quelites: alimentos ricos en nutrimentos y compuestos antioxidantes}

Los quelites son capaces de adaptarse a climas secos, donde el agua y los nutrientes escasean y donde su producción no requiere el uso de fertilizantes o riego frecuente ${ }^{4,6,12,17}$. De esta manera, al considerarse plantas tolerantes a condiciones adversas, sugieren que la sequía, salinidad y demás factores han sido una ventaja para esta variedad de especies vegetales, promoviendo mecanismos de protección frente al estrés ${ }^{20}$; observándose en la acumulación de aminoácidos, síntesis de compuestos fenólicos y polisacáridos, por mencionar algunos, y otorgándoles capacidades antioxidantes y perfiles nutrimentales excelentes (Tablas 1 y 3).

Las plantas del género Amaranthus spp. (Amaranthus hybridus, Amaranthus hypochondriacus y Amaranthus tricolor), Chenopodium spp. y la especie de Portulaca oleracea L. tienen la capacidad de crecer a pesar de factores limitantes ${ }^{20,21,22}$. Se ha reportado que Amaranthus spp. al someterse a largos períodos de estrés (sequías) mostró la capacidad de acumular prolina, aminoácido que permite a la planta activar una osmoregulación, previniendo el estrés hiperosmótico ${ }^{20}$; mientras que Portulaca oleracea ${ }^{22}$ y Chenopodium spp. ${ }^{21}$ incrementaron la producción de antioxidantes y metabolitos que le permiten sobrevivir y reducir el daño celular, resistiendo de esta manera el estrés abiótico. Estas plantas adicionalmente, han reportado ser fuentes de proteína, fibra dietética, nutrimentos inorgánicos (Fe, Mn, Zn, B, P, Ca, Mg, K), ácidos fenólicos (cafeico, gálico, clorogénico, cumárico, ferúlico), flavonoides (rutina, quercetina), carotenoides, clorofila y vitaminas (A,C); los cuales se presentan en diversas especies de quelites procedentes de México ${ }^{8,9,23}$, África (Sudáfrica) ${ }^{20}$ y Norteamérica ${ }^{16}$, entre otros países (Tabla 1). En la tabla 1 se puede observar que en relación con las especies mexicanas de Amaranthus spp. se presenta mayor contenido de proteína (3-5g/100g de peso fresco $)^{8,9,23}$. Los compuestos fenólicos que han sido identificados varían de acuerdo a la zona geográfica de la especie; por ejemplo, la especie de Amaranthus hybridus procedente del estado de Hidalgo, México, presentó ácido gálico, clorogénico, $p$-cumárico, ferúlico y florizidina ${ }^{9}$; mientras que la especie de Amaranthus hypochondriacus L. proveniente de Puebla, México, presentó 2-metoxi-4 vinilfenol $^{23}$, compuestos de importancia nutrimental que no habían sido reportados en otras especies del género Amaranthus spp. procedentes de México y otros países. Por otra parte, las plantas pertenecientes al género Chenopodium spp., han desplegado de igual manera una interesante gama de compuestos nutrimentales en sus estructuras. Las hojas y partes aéreas de este género de plantas son una excelente fuente de nutrimentos inorgánicos como $\mathrm{Ca}, \mathrm{K}, \mathrm{Mg}$ y $\mathrm{Zn}^{24}$, y ácidos fenólicos y flavonoides como quercetina, kaempferol, ácido vanílico y rutina ${ }^{25,26}$, convirtiéndose en un alimento óptimo para la nutrición humana (Tabla 2). Especies mexicanas de este género procedentes de Hidalgo, México ${ }^{9}$ y la Sierra Norte de Puebla, México ${ }^{8}$ (Chenopodium berlandieri L.) fueron identificadas como fuentes importantes de proteína (2-5 g/100g de peso fresco), fibra dietética $(2-4 \mathrm{~g} / 100 \mathrm{~g}$ de peso fresco), vitamina $\mathrm{A}$, clorofila ( $6.6 \mathrm{mg} / \mathrm{g}$ de peso fresco) así como un alto contenido de macroelementos (Fe, Cu, Mn, Zn, B) y microelementos $(\mathrm{P}, \mathrm{Ca}, \mathrm{Mg}, \mathrm{K})$; concentraciones que resultaron mayores al compararse con valores de referencia de especies procedentes de otros países, como Chenopodium album $\mathrm{L}^{27,28}$. Una investigación reciente, identificó y cuantificó en Chenopodium berlandieri L. ${ }^{9}$ compuestos fenólicos como ácido gálico, clorogénico, vainillínico, ferúlico, siríngico, floretina, florizidina y mirecetina, los cuales no habían sido reportados en esta especie. Cabe mencionar que la información relacionada a la especie de Chenopodium berlandieri L. que se desarrolla en México aún es escasa, por lo que en la Tabla 2 se incluyen el aporte nutrimental y funcional de este género que se utiliza en otros países. En la literatura se han reportado diversos componentes encontrados en las partes aéreas de la familia Portulacaceae (Tabla 3). De esta familia, una de las especies de gran importancia en México es la verdolaga, considerado como un alimento accesible, económico y como una buena fuente de antioxidantes como betacarotenos, vitamina $\mathrm{C}$, ácidos cumárico y cafeico ${ }^{29}$, nutrimentos inorgánicos como $\mathrm{K}, \mathrm{Mg}$ y $\mathrm{Zn}^{30,31}$, entre otros componentes; desplegando un excelente valor nutrimental y beneficio antioxidante. Recientemente un estudio realizado en Portulaca oleracea L. nativa de México ${ }^{9}$, mostró que además de los nutrimentos ya reportados en la verdolaga (ácidos grasos omega 3 y 6, alcaloides, nutrimentos inorgánicos) también presenta mirecetina al igual que Chenopodium berlandieri L. ${ }^{9}$, un flavonoide natural mayoritario en bayas y vino tinto con actividad antioxidante y antitumoral ${ }^{32}$, el cual no había sido observado en especies mexicanas de verdolaga y quelite cenizo. Como se observa en las tablas 1 a 3 los quelites son una excelente fuente de compuestos bioactivos por lo 
Tabla 1. Compuestos bioactivos y nutrimentales identificados en la familia Amaranthaceae, género Amaranthus.

\begin{tabular}{|c|c|c|c|}
\hline Especie & Estructura evaluada & Compuestos identificados & Referencia \\
\hline $\begin{array}{l}\text { Amaranthus hypochondriacus } \\
\text { Amaranthus hybridus }\end{array}$ & Hojas & $\begin{array}{c}\text { Prolina } \\
(380-455 \mu \mathrm{mol} g / \mathrm{ps})\end{array}$ & [20] \\
\hline Amaranthus spp. & Hojas & $\begin{array}{c}\text { Proteína (17,5-30,3\% de ms) } \\
\text { Lisina ( } 5 \% \text { de ms); Calcio }(1,7 \pm 0,04 \text { g/100 g) } \\
\text { Hierro }(1234 \pm 50,0 \text { mg/kg); Zinc }(791,7 \pm 28,9 \text { mg/kg) }\end{array}$ & {$[50,51]$} \\
\hline $\begin{array}{l}\text { Amaranthus acanthochiton } \\
\text { Amaranthus deflexus } \\
\text { Amaranthus viridis }\end{array}$ & Hojas & $\begin{array}{l}\text { Nutrimentos inorgánicos (Fe, Ca, Mg, Ni, Zn, Mn, Mo) } \\
\text { Vitamina C (0,7-1,4 mg AA/g pf); Fenoles totales (3,2-5,5 mg } \\
\text { EAG/g pf); Flavonoides (0,98-4,1 mg EC/g pf) }\end{array}$ & [16] \\
\hline $\begin{array}{l}\text { Amaranthus hypochondriacus } \\
\text { Amaranthus caudatus } \\
\text { Amaranthus cruentus }\end{array}$ & $\begin{array}{l}\text { Semillas } \\
\text { Hojas } \\
\text { Flores }\end{array}$ & $\begin{array}{c}\text { Betaninas totales }(0,07-0,96 \mathrm{mg} / 100 \mathrm{~g} \mathrm{ps}) \\
\text { Amarantina }(0,04-0,44 \mathrm{mg} / 100 \mathrm{~g} \mathrm{ps}) \\
\text { Isoamarantina }(0,02-0,28 \mathrm{mg} / 100 \mathrm{~g} \mathrm{ps}) \\
\text { Betaninas totales }(16,90-20,93 \mathrm{mg} / 100 \mathrm{~g} \mathrm{ps}) \\
\text { Amarantina }(7,75-9,67 \mathrm{mg} / 100 \mathrm{~g} \mathrm{ps}) \\
\text { Isoamarantina }(5,13-6,38 \mathrm{mg} / 100 \mathrm{~g} \mathrm{ps}) \\
\text { Betaninas totales }(0,95-6,02 \mathrm{mg} / 100 \mathrm{~g} \mathrm{ps}) \\
\text { Amarantina }(0,45-2,76 \mathrm{mg} / 100 \mathrm{~g} \mathrm{ps}) \\
\text { Isoamarantina }(0,28-1,80 \mathrm{mg} / 100 \mathrm{~g} \mathrm{ps})\end{array}$ & [33] \\
\hline Amaranthus hypochondriacus L. & $\begin{array}{l}\text { Hojas } \\
\text { Semillas }\end{array}$ & $\begin{array}{l}\text { Fibra cruda }(2,60 \%) ; \text { Proteína }(3,78 \%) \\
\text { Fibra cruda }(5,80 \%) ; \text { Proteína }(16,73 \%)\end{array}$ & [23] \\
\hline Amaranthus caudatus & Semillas & Ácido protocatecuico $(13,6$ mol/100 g ps) & {$[52]$} \\
\hline Amaranthus hypochondriacus & Semillas & $\begin{array}{l}\text { Ácidos fenólicos y flavonoides (Isoquercitina, } \\
\text { nicoriflorina, rutina, ácido 4-hidroxibenzoico, } \\
\text { ácido siríngico, ácido vanílico) }\end{array}$ & [53] \\
\hline Amaranthus cruentus & Hojas & $\begin{array}{l}\text { Aminoácidos (alanina, leucina, isoleucina, valina, } \\
\text { glicina, aspartato, glutamato, metionina) } \\
\text { Nutrimentos inorgánicos }(\mathrm{Ca}, \mathrm{Fe}, \mathrm{Mg}, \mathrm{K}, \mathrm{Zn})\end{array}$ & [35] \\
\hline
\end{tabular}

pf: peso fresco; ps: peso seco; ms: materia seca; AA: ácido ascórbico; EAG: equivalentes de ácido gálico; EC: equivalentes de catequina.

cual se pueden considerar como alimentos que aportan compuestos antioxidantes de manera exógena al cuerpo humano ${ }^{33,34}$. Los quelites también presentan otros compuestos de interés para la nutrición humana, como aminoácidos esenciales y no esenciales ${ }^{35}$, ácidos grasos poliinsaturados ${ }^{36}$ y fibra dietética ${ }^{8,9}$ los cuales son de importancia en estratos sociales marginados, donde las fuentes por excelencia de estos nutrimentos no son accesibles.

Propiedades medicinales y beneficios a la salud humana Los quelites, son recomendados como una fuente de 
Tabla 2. Compuestos bioactivos y nutrimentales identificados en hojas de la familia Amaranthaceae, género Chenopodium.

\begin{tabular}{|c|c|c|}
\hline Especie & Compuestos identificados & Referencia \\
\hline Chenopodium spp. & $\begin{array}{c}\text { Por cada } 100 \mathrm{~g} \text { peso seco: } \\
\mathrm{K}(6048.39 \mathrm{mg}) ; \mathrm{Ca}(1154.30 \mathrm{mg}) \\
\mathrm{Na}(958,04 \mathrm{mg}) ; \mathrm{Fe}(83,60 \mathrm{mg}) ; \mathrm{Mg}(81,29 \mathrm{mg})\end{array}$ & [24] \\
\hline $\begin{array}{l}\text { Chenopodium album L. } \\
\text { Chenopodium spp. }\end{array}$ & $\begin{array}{c}\text { Proteína }(3,63-4,51 \text { g/100g pf); vitamina C (11,15-26,12 g/100g ps) } \\
\text { clorofila a (0,998 mg/100g pf); clorofila b (0,297 mg/100g pf) } \\
\text { carotenoides }(0,98-1,40 \text { mg/100g pf })\end{array}$ & {$[27,28]$} \\
\hline $\begin{array}{l}\text { Chenopodium foliosum Asch } \\
\text { Chenopodium } \\
\text { bonus-henricus L. }\end{array}$ & $\begin{array}{c}\text { Flavonoides: } \\
\text { patuletina } \\
\text { espinacetina } \\
\text { 6-Metoxikanferol }\end{array}$ & {$[34,44]$} \\
\hline Chenopodium spp. & $\begin{array}{c}\text { Polifenoles: } \\
\text { parte herbácea }(3,36 \mathrm{mg} / \mathrm{g} \mathrm{ps}) ; \text { semillas }(3,87 \mathrm{mg} / \mathrm{g} \mathrm{ps}) \\
\text { raíz }(1,52 \mathrm{mg} / \mathrm{g} \mathrm{ps}) .\end{array}$ & [26] \\
\hline Chenopodium album & $\begin{array}{l}\text { Fenoles totales (14,56-42,00 mg EAG/g extracto) } \\
\text { Flavonoides }(1,498-6,373 \mathrm{mg} \text { ER/g extracto) } \\
\text { Flavonona }(0,671-1,037 \mathrm{EN} / \mathrm{g} \text { extracto })\end{array}$ & [54] \\
\hline $\begin{array}{l}\text { Chenopodium formosanum } \\
\text { Koidz. (djulis) }\end{array}$ & $\begin{array}{c}\text { Ácidos fenólicos de } 11,5 \mu \mathrm{g} / \mathrm{g} \text { de extracto } \\
\text { (caffeoil-putrescina (2)) a } 1855.3 \mu \mathrm{g} / \mathrm{g} \\
\text { (pentosida ácida hidroxilfenilacética 3) } \\
\text { Flavonoides de } 19,93 \mu \mathrm{g} / \mathrm{g} \text { de extracto (quercetina-3-O- (cumaril)) } \\
\text {-rutinosida-pentosida (1)) a } 257,3 \pm 2,05 \mu \mathrm{g} / \mathrm{g} \text { (rutina-O-pentosida (2)) }\end{array}$ & [25] \\
\hline
\end{tabular}

pf: peso fresco; ps: peso seco; ms: materia seca; EAG: equivalentes de ácido gálico; ER: equivalentes de rutina; EN: equivalente de naringina.

proteína, nutrimentos inorgánicos y otros micronutrimentos esenciales en la dieta ${ }^{6}$. Los tallos, flores y hojas son una fuente alternativa por su aporte de diversos compuestos bioactivos, los cuales desempeñan funciones antioxidantes y preventivas en diversas fisiopatologías cardiovasculares ${ }^{37}$ y procesos cancerígenos ${ }^{38}$. Aun es limitada la literatura que existe sobre el consumo de quelites en México y su efecto a la salud humana, sin embargo, se han estudiado estos mismos géneros, pero de diferente especie en otras partes del mundo y sus efectos biológicos se pueden observar en las Tablas 4 a 6. Algunas especies de Amaranthus spp. han mostrado su efectividad como agentes antihelmínticos (Amaranthus viridis $^{39}$ y protectores en contra de la aflatoxina B1 (AFB1)

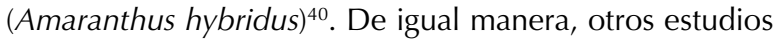
reportados en Amaranthus caudatus ${ }^{41}$, mostraron cambios positivos con dosis de $200-400 \mathrm{mg} / \mathrm{kg} /$ día de esta planta, en ratas con daño hepático, donde se observó cambios en enzimas séricas, MDA (malondialdehído) y otros parámetros enzimáticos. Este estudio observó la normalización de los valores bioquímicos para AST (aspartato aminotransferasa), ALT (alanina aminotransferasa) y BT (billirubina total); en el caso de la peroxidación, los valores de MDA se vieron reducidos, mientras que la actividad de GSH (glutatión reducido) y CAT (catalasa) se incrementaron. De igual manera se realizó un estudio histopatológico encontrando reducción en la degeneración de hepatocitos, lo que sugiere una actividad 
Tabla 3. Compuestos bioactivos y nutrimentales identificados en la familia Portulacaceae.

\section{Especie}

Talinum triangulare

Portulaca oleracea L.

\section{Compuestos identificados}

Acrilamidas; Ácido propanoico

Feofitinas; (Ficusclorina, taliclorina, feofitina b peroxilactona)
Referencia

[46]

Portulacanonas $(A-D)$

Fenoles totales $(0,96-9,12 \mathrm{mg}$ EAG/g ps)

Flavonoides totales (0,13-1,44 ER/g ps); Carotenoides totales (0,52-5,64 E $\beta$ )

Nutrimentos inorgánicos:

$\mathrm{P}$ (5,08-14,18 g/kg bs); K (266-656 g/kg bs); Ca (35-104,2 g/kg bs); Mg (40,8-101,4 g/kg bs)

Fe (1,86-11,1 g/kg bs); Zn (0,62-1,48 g/kg bs); Mn (0,14-1,64 g/kg bs)

$$
\text { Alcaloides: (Oleraceínas A, B, C, D, E) }
$$

Omega 3 (4,06 mg/g pf); omega 6 (0,89 mg/g pf)

$\beta$-carotenos (43,5 mg/100g ps); $\alpha$-tocoferol (170 mg/100g ps)

ácido ascórbico (451 mg/100g ps); glutatión (14,81 mg/100g pf)

Compuestos fenólicos (316,35 mg/kg ps): ácido cumárico, cafeico, ferúlico, rosmarínico.

Alcaloides:

(aurantiamideacetato, aurantiamida, 1,5-dimetil-6-fenil-1,6,3,4,-tetrahidro-1,2,4- 2(1H)-triazina, trollisina, ciclo (L- tirosinil-L - tirosinil), 3,5-bis (3-metoxi, 4 -hidroxifenil)-5,6- dihidro,2(1H)piridinona, $\mathrm{N}$-feruloil normetaneferina, $\mathrm{N}$-trans- feruloil tiramina.

Polisacáridos; Glucósidos; Monoterpenos

Portulacerebrósido A

Talinum

triangulare (Jacq.)

Willd.
Betacianinas:

flores $(4,136 \mathrm{mg} / \mathrm{g} \mathrm{pf})$; tallo $(2,232 \mathrm{mg} / \mathrm{g} \mathrm{pf})$ hojas $(0,065 \mathrm{mg} / \mathrm{g}$ pf).

Betaxantinas:

hojas $(1,032 \mathrm{mg} / \mathrm{g}$ pf); flores $(0,857 \mathrm{mg} / \mathrm{g} \mathrm{pf})$

tallos (0,546 mg/g pf); Betalaínas totales: 4,993 (mg/g pf)

pf: peso fresco; ps: peso seco; bs: base seca; EAG: equivalentes de ácido gálico; ER: equivalentes de rutina; Eß: equivalentes de $\beta$-caroteno.

hepatoprotectora (Tabla 5). El género Chenopodium spp. por otro lado, ha sido bien descrito por sus numerosas propiedades medicinales en la medicina tradicional; la literatura ha confirmado actividades antibacterianas, y anticancerígenas (Chenopodium quinoa) ${ }^{42,43}$; por otra parte, otros ensayos han demostrado que Chenopoduim bonushenricus L. ${ }^{44}$ tiene un efecto positivo a dosis de $100 \mathrm{mg} / \mathrm{kg} /$ día en ratas con daño hepático inducidas con tetracloruro de carbono, demostrando una reducción en MDA, aumento en la actividad de enzimas, SOD (superóxido dismutasa), GPx (glutatión peroxidasa), e incremento de la viabilidad celular comparado con el agente tóxico; exhibiendo el potencial efecto hepatoprotector y antioxidante de Chenopodium spp. Otro estudio realizado en Chenopodium quino ${ }^{43}$, evaluó la actividad de las hojas a dosis de 0.189-1.89 mg/ $\mathrm{mL}$ en líneas celulares de cáncer de próstata; observando una disminución de la peroxidación lipídica y disminución en el crecimiento de líneas celulares cancerígenas. En cuanto a Portulaca oleracea, se ha reportado efectos inmunoestimulantes ${ }^{45}$, antiparasitarios ${ }^{46}$, propiedades antioxidantes y de quelación de iones metálicos ${ }^{31}$ y efectos neuroprotectores en enfermedades neurovasculares isquémicas 
Tabla 4. Efectos biológicos reportados en plantas de la familia Amaranthaceae, género Amaranthus.

\begin{tabular}{|c|c|c|c|c|}
\hline Especie & $\begin{array}{l}\text { Estructura } \\
\text { analizada }\end{array}$ & $\begin{array}{l}\text { Tipo de } \\
\text { ensayo }\end{array}$ & Efecto biológico & Referencia \\
\hline Amaranthus hybridus & Hojas & $\begin{array}{l}\text { Ensayo } \\
\text { in vitro }\end{array}$ & $\begin{array}{l}\text { Disminución del daño del DNA } \\
\text { ocasionado por micotoxinas (aflatoxina B1) }\end{array}$ & {$[40]$} \\
\hline $\begin{array}{l}\text { Amaranthus } \\
\text { hypochondriacus L. }\end{array}$ & Hojas & $\begin{array}{l}\text { Ensayos } \\
\text { in vitro }\end{array}$ & $\begin{array}{l}\text { Aumento de la capacidad antioxidante } \\
\text { Propiedades nutrimentales (proteína) }\end{array}$ & [23] \\
\hline $\begin{array}{l}\text { Amaranthus } \\
\text { caudatus }\end{array}$ & Hojas & $\begin{array}{l}\text { Ensayos } \\
\text { preclínicos }\end{array}$ & $\begin{array}{c}\text { Efectos antihiperlipidémicos } \\
\text { Disminución de niveles séricos de colesterol } \\
\text { y triglicéridos }\end{array}$ & [37] \\
\hline $\begin{array}{l}\text { Amaranthus viridis } \\
\text { Amaranthus spinosus }\end{array}$ & & & Incremento de HDL (lipoproteínas de alta densidad) & \\
\hline $\begin{array}{l}\text { Amaranthus } \\
\text { Caudatus L. }\end{array}$ & Hojas & $\begin{array}{l}\text { Ensayos } \\
\text { preclínicos }\end{array}$ & $\begin{array}{c}\text { Antinociceptivo } \\
\text { Antipirético } \\
\text { Actividad hepatoprotectora } \\
\text { Prevención del incremento del malondialdehído (MDA), } \\
\text { glutatión (GSH), catalasa (CAT) y niveles de tioles totales (TT). }\end{array}$ & {$[41,59]$} \\
\hline \multirow[t]{5}{*}{ Amaranthus viridis } & $\begin{array}{l}\text { Partes } \\
\text { aéreas }\end{array}$ & $\begin{array}{l}\text { Ensayos } \\
\text { in vitro }\end{array}$ & Actividad antihelmíntica. & [39] \\
\hline & & $\begin{array}{l}\text { Ensayos } \\
\text { preclínicos }\end{array}$ & $\begin{array}{l}\text { Actividad hepatoprotectora } \\
\text { Efectos antidiabéticos } \\
\text { Disminución de glucosa en sangre } \\
\text { Efectos antihiperlipidémicos } \\
\text { Estabilidad del perfil lipídico }\end{array}$ & $\begin{array}{l}{[60]} \\
{[61]}\end{array}$ \\
\hline & & & $\begin{array}{c}\text { Inhibición de la proliferación de células cancerígenas } \\
\text { Células-B linfoma, células -B hibridoma y macrófagos } \\
\text { de líneas celulares cancerígenas. } \\
\text { Actividad antifúngica } \\
\text { Fitopatógenos }\end{array}$ & [62] \\
\hline & Semillas & $\begin{array}{l}\text { Ensayos } \\
\text { in vitro }\end{array}$ & $\begin{array}{c}\text { Actividad antiviral } \\
\text { (Virus del sarampión en línea celular del carcinoma } \\
\text { epidermoide humano (HEP-2)) } \\
\text { Prevención de la replicación del virus } \\
\text { Atenuación del virus después de la penetración a la célula } \\
\text { Bloqueo de receptores virales }\end{array}$ & 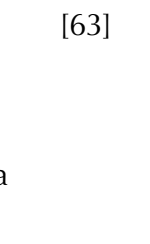 \\
\hline & Hojas & $\begin{array}{l}\text { Ensayos } \\
\text { in vitro }\end{array}$ & $\begin{array}{c}\text { Efectos antiinflamatorios } \\
\text { Inhibición de la hialuronidasa, lipooxigenasa, } \\
\text { y xantina oxidasa. } \\
\text { Inhibición de HMG-CoA reductasa } \\
\text { (3-hidroxi-3-metil-glutaril-CoA reductasa) } \\
\text { Actividad antioxidante }\end{array}$ & [64] \\
\hline Amaranthus spinosus & Hojas & $\begin{array}{l}\text { Ensayos } \\
\text { preclínicos }\end{array}$ & $\begin{array}{l}\text { Efectos quimioprotectores } \\
\text { MDA, GSH, CAT, TT } \\
\text { Actividad antioxidante }\end{array}$ & [38] \\
\hline
\end{tabular}


Tabla 5. Efectos biológicos reportados en plantas de la familia Amaranthaceae, género Chenopodium.

\begin{tabular}{|c|c|c|c|c|}
\hline Especie & $\begin{array}{l}\text { Estructura } \\
\text { analizada }\end{array}$ & $\begin{array}{l}\text { Tipo de } \\
\text { ensayo }\end{array}$ & Efecto biológico & Referencia \\
\hline $\begin{array}{l}\text { Chenopodium album } \\
\text { Chenopodium hybridum } \\
\text { Chenopodium rubrum } \\
\text { Chenopodium urbicum }\end{array}$ & $\begin{array}{l}\text { Partes aéreas } \\
\text { Semillas }\end{array}$ & $\begin{array}{l}\text { Ensayos } \\
\text { In vitro }\end{array}$ & $\begin{array}{c}\text { Efectos antitumorales } \\
\text { Disminución de proliferación de células en } \\
\text { carcinoma de ovario (Células TOV-112D). } \\
\text { Propiedades antioxidantes }\end{array}$ & [26] \\
\hline $\begin{array}{l}\text { Chenopodium incisum Poir } \\
\text { Chenopodium murale L. } \\
\text { Chenopodium ambrosioides L }\end{array}$ & $\begin{array}{l}\text { Partes aéreas } \\
\text { Toda la planta } \\
\text { Raíz }\end{array}$ & $\begin{array}{l}\text { Ensayos } \\
\text { In vitro }\end{array}$ & $\begin{array}{l}\text { Actividad antibacteriana (Escherichia coli, } \\
\text { Staphylococcus aureus, Bacillus subtilis, } \\
\text { Shigella sonnei, Shigella flexneri) }\end{array}$ & [42] \\
\hline Chenopodium ambrosioides L. & Hojas & $\begin{array}{l}\text { Ensayos } \\
\text { In vitro }\end{array}$ & $\begin{array}{c}\text { Actividad antibacteriana } \\
\text { (Sthapylococcus aureus IS-58) }\end{array}$ & [65] \\
\hline Chenopodium foliosum Asch & $\begin{array}{l}\text { Partes s } \\
\text { aérea }\end{array}$ & $\begin{array}{l}\text { Ensayos } \\
\text { In vitro }\end{array}$ & $\begin{array}{c}\text { Actividad antioxidante } \\
\text { Propiedades anticancerígenas } \\
\text { Propiedades inmunoestimulantes }\end{array}$ & [34] \\
\hline $\begin{array}{l}\text { Chenopodium album } \\
\text { Chenopodium bonus-henricus L. }\end{array}$ & $\begin{array}{l}\text { Partes } \\
\text { aéreas }\end{array}$ & $\begin{array}{l}\text { Ensayos } \\
\text { preclínicos }\end{array}$ & $\begin{array}{l}\text { Propiedades antianémicas } \\
\text { Propiedades expectorantes } \\
\text { Propiedades antiasmáticas } \\
\text { Propiedades antihelmínticas }\end{array}$ & [66] \\
\hline Chenopodium album Linn. & Hojas & $\begin{array}{l}\text { Ensayos } \\
\text { preclínicos }\end{array}$ & $\begin{array}{l}\text { Agente Antilitiasis } \\
\text { Inhibición del crecimiento y tamaño } \\
\text { de cristales (oxalatos) } \\
\text { Menor retención de cristales en túbulos } \\
\text { y prevención de daño necrótico en riñón. } \\
\text { Tratamiento en enfermedades urinarias y renales }\end{array}$ & [67] \\
\hline Chenopodium bonus-henricus L. & $\begin{array}{l}\text { Partes } \\
\text { aéreas }\end{array}$ & $\begin{array}{l}\text { Ensayos } \\
\text { in vitro }\end{array}$ & $\begin{array}{l}\text { Actividades hepatoprotectoras y antioxidantes. } \\
\text { Reducción del daño celular ocasionado por } \\
\text { CCl4 (cloruro de carbono) en hepatocitos. } \\
\text { Preservación de la viabilidad celular y } \\
\text { niveles de GSH (glutatión) } \\
\text { Reducción del daño lipídico }\end{array}$ & [44] \\
\hline Chenopodium quinoa & Hojas & $\begin{array}{l}\text { Ensayos } \\
\text { In vitro }\end{array}$ & $\begin{array}{c}\text { Efectos quimiopreventivos y anticarcinogénicos } \\
\text { Actividades quelantes } \\
\text { Actividades antioxidantes } \\
\text { Disminución de la oxidación lipídica } \\
\text { Disminución de la proliferación y movilidad } \\
\text { en células de cáncer de próstata. }\end{array}$ & [43] \\
\hline Chenopodium spp. & $\begin{array}{l}\text { Hojas } \\
\text { Partes } \\
\text { aéreas } \\
\text { Frutos }\end{array}$ & $\begin{array}{l}\text { Ensayos } \\
\text { In vitro }\end{array}$ & $\begin{array}{c}\text { Efectos inmunomoduladores } \\
\text { Efectos hipotensivos } \\
\text { Hemaglutinativos } \\
\text { Actividad antihelmíntica } \\
\text { Actividad antibacteriana } \\
\text { (Klebsiella pneumoniae, Pseudomonas } \\
\text { aeruginosa, Candida albicans) } \\
\text { Actividad antifúngica } \\
\text { Actividad antiviral }\end{array}$ & [68] \\
\hline Chenopodium ambrosioides & Semillas & $\begin{array}{l}\text { Ensayos } \\
\text { preclínicos }\end{array}$ & $\begin{array}{l}\text { Actividad antileishmania } \\
\text { (L. amazonensis) }\end{array}$ & [69] \\
\hline
\end{tabular}


Tabla 6. Efectos biológicos reportados en las partes aéreas de plantas de la familia Portulacaceae.

\begin{tabular}{|c|c|c|c|}
\hline Especie & Tipo de ensayo & Efecto biológico & Referencias \\
\hline \multirow[t]{4}{*}{ Portulaca oleracea } & Ensayos preclínicos & $\begin{array}{c}\text { Antioxidante } \\
\text { Disminución del } \mathrm{H}_{2} \mathrm{O}_{2} \text { (peróxido de hidrógeno) } \\
\text { producto del estrés oxidativo. } \\
\text { Protección contra agentes neurotóxicos } \\
\text { ambientales (Rotenona) }\end{array}$ & [49] \\
\hline & & $\begin{array}{l}\text { Efectos neuroprotectores } \\
\text { Estimula la expresión endógena de eritropoyetina, } \\
\text { estabilizando la expresión de HIF-1 } \alpha \text { (subunidad alfa del } \\
\text { factor } 1 \text { inducible por hipoxia o factor inducible por hipoxia) } \\
\text { Disminuye la actividad de la caspasa } 3 \text { en neuronas. } \\
\text { Incrementa la viabilidad de la neurona. } \\
\text { Disminuye los daños causados por condiciones hipóxicas. }\end{array}$ & [47] \\
\hline & $\begin{array}{l}\text { Ensayos } \\
\text { In vitro } \\
\text { Ensayos preclínicos }\end{array}$ & $\begin{array}{c}\text { Actividades antidiabéticas } \\
\text { Incremento de la captación extracelular de glucosa } \\
\text { Mejoramiento de la secreción de insulina } \\
\text { Actividad antioxidante }\end{array}$ & [70] \\
\hline & Ensayos preclínicos & $\begin{array}{c}\text { Efectos antitumorales } \\
\text { Propiedades inmunoestimulantes } \\
\text { Inhibe la proliferación de células tumorales } \\
\text { Incremento de células blancas, linfocitos CD4+T } \\
\text { Disminución de la aspartato transaminasa sérica (AST), } \\
\text { alanina transaminasa (ALT), nitrógeno ureico (BUN) y creatinina. }\end{array}$ & [45] \\
\hline $\begin{array}{l}\text { Portulaca werdermannii } \\
\text { Portulaca hirsutissima }\end{array}$ & $\begin{array}{l}\text { Ensayos } \\
\text { In vitro }\end{array}$ & $\begin{array}{l}\text { Actividad antileshmania } \\
\text { (Leishmania amazonensis) } \\
\text { Actividad inmunomoduladora }\end{array}$ & [46] \\
\hline \multirow[t]{2}{*}{$\begin{array}{l}\text { Talinum triangulare } \\
\text { (Jacq.) Willd }\end{array}$} & $\begin{array}{l}\text { Ensayos } \\
\text { In vitro }\end{array}$ & $\begin{array}{l}\text { Efecto de estabilidad de radicales libres. } \\
\text { Elevada actividad antioxidante } \\
\text { Actividad de quelación de iones metálicos } \\
\text { iones de Fe }\end{array}$ & {$[31]$} \\
\hline & $\begin{array}{l}\text { Ensayos } \\
\text { In vitro }\end{array}$ & $\begin{array}{c}\text { Actividad antioxidante } \\
\text { Disminución de la peroxidación lipídica. }\end{array}$ & [29] \\
\hline Portulaca oleracea & Ensayos preclínicos & $\begin{array}{c}\text { Efectos neuroprotectores } \\
\text { Disminución del daño oxidativo ocasionado por } \\
\text { D-galactosa en procesos de senescencia } \\
\text { Mejoramiento de memoria y viabilidad de actividad neuronal } \\
\text { Incremento de la actividad de enzimas antioxidantes } \\
\text { (superóxido dismutasa (SOD), catalasa (CAT), glutatión } \\
\text { peroxidasa (GPx) y glutatión reductasa (GR)) }\end{array}$ & [48] \\
\hline
\end{tabular}

hipóxicas (incremento de la viabilidad de la neurona) ${ }^{47}$ y disminución del daño oxidativo ocasionado por D-galactosa en procesos de senescencia ${ }^{48}$. Adicionalmente un estudio en ratas inducidas a estrés oxidativo por rotenona exploró el efecto de la verdolaga de reducir el daño de este contaminante, al administrar dosis de jugo de verdolaga $(1.5 \mathrm{~mL} / \mathrm{kg} / \mathrm{día})^{49}$; donde se demostró que después de la administración de la planta y de la exposición al insecticida se encontró un incremento en la actividad de Na+-K+-ATPasa en cuerpo estriado, disminución de proteínas carboniladas en tejido neural, elevación de GSH en mesencéfalo, e incremento de la actividad de GPx y CAT; confiriendo a esta planta 
propiedades neuroprotectoras en daños oxidativos inducidos por contaminantes antropogénicos. Otro estudio usando Portulaca olerace ${ }^{45}$ con dosis de $25-100 \mathrm{mg} / \mathrm{kg} / \mathrm{día}$ en ratones con sarcomas, observó una inhibición del crecimiento del sarcoma, reducción del tamaño del tumor, incremento del peso de los órganos relacionados con el sistema inmune, estimulación de linfocitos en vaso, estimulación de células T helper para CD4 y la normalización de parámetros hematológicos y bioquímicos, demostrando así propiedades antitumorales de esta planta.

\section{CONCLUSIONES}

Actualmente México experimenta una dualidad en su situación alimentaria, por un lado, una alta producción de alimentos, pero con alta prevalencia de hogares con inseguridad alimentaria, cuyas cifras siguen en aumento en especial en poblaciones con bajo poder adquisitivo. La disponibilidad e insuficiencia de alimentos repercuten directamente con la ingesta diaria de nutrimentos y por ende en el estado nutricio de las familias. Se ha confirmado que los quelites, son alimentos consumidos y aceptados gastronómicamente, económicos y lo que es más importante, ofrecen una amplia variedad de fitoquímicos y nutrimentos esenciales en la salud humana. Es por ello, que la presente revisión destaca la importancia cultural y nutrimental de los quelites en la alimentación mexicana, por lo que se recomienda que estas especies se reincorporen dentro de la dieta y que se utilicen como una estrategia para complementar en conjunto con una dieta correcta las demandas nutrimentales de los diferentes estratos sociales, contribuyendo a la seguridad alimentaria de un país en constante cambio económico, de salud y climático.

Agradecimientos. Este trabajo fue apoyado por CONACYT (beca no. 273250), que permitió la realización de esta revisión derivada del trabajo experimental en poblaciones marginadas de México, para la promoción de estrategias encaminadas a la seguridad alimentaria en grupos vulnerables.

\section{BIBLIOGRAFÍA}

1. Agro-Food and fishing information service (SIAP). Agro-Food Atlas: Mexico, Agro-Food sector in stadistics. SIAP., Mexico city, 2017.

2. Gutiérrez J, Rivera-Dommarco J, Shamah-Levy T, VillalpandoHernández S, Franco A, Cuevas-Nasu L, Romero-Martínez M, Hernández-Ávila M. National Health and Nutrition Survey 2012: National results. Public Health National Institute., Mexico city, 2012.

3. Castro-Lara D, Basurto-Peña F, Mera-Ovando L, Bye $R$. Quelites traditional food in Mexico. Chapingo Autonomous University., Mexico state, 2011.

4. Mera-Ovando LM, Castro-Lara D, Bye R. Low-valued plant species: an alternative for food security. NAM-SNICS-SINAREFI., Mexico city, 2011

5. Basurto F. The quelites of Mexico: species of current use, in low-valued vegetable species: an alternative for food security. UNAM-SNICS-SINAREFI., Mexico city, 2011.

6. Linares $M$, Bye $R$. The under-utilized species of the milpa. RDU 2015; 16(5): 22.
7. Castro-Lara D, Basurto-Peña F, Mera-Ovando L, Bye $R$. Quelites, millenary tradition in Mexico. Chapingo Autonomous University., Mexico state, 2011.

8. Mera-Ovando L, Alvarado-Flores R, Basurto-Peña $F$, ByeBoettler R, Castro-Lara D, Evangelista V, Mapes-Sánchez C, Martínez-Alfaro M, Molina N, Saldivar J. Quelites, I eat a taco: Experience in nutrition education. Revista Jard Bot Nac 2003; 24(1-2): 45-49.

9. Santiago-Saenz $Y$, Hernández-Fuentes A, Monroy-Torres $R$, Cariño-Cortés $R$, Jiménez-Alvarado R. Physicochemical, nutritional and antioxidant characterization of three vegetables (Amaranthus hybridus L., Chenopodium berlandieri L., Portulaca oleracea L.) as potential sources of phytochemicals and bioactive compounds. J Food Meas Charact 2018; 12(4): 2855-2864.

10. Velázquez-Ibarra A, Covarrubias-Prieto J, Ramírez-Pimentel J, Aguirre-Mancilla C, Iturriaga de la Fuente G, Raya-Pérez J. Nutritional quality of Mexican quelites (Green leafy). Ciencia y Tecnol Agrop 2016; 4(2): 1-9.

11. Figueroa P. Measurement of Food and Nutrition security. Federal University of Pernambuco., Brazil, 2005.

12. Bye $R$, Linares $E$. The quelites, edible plants of Mexico: a reflection on cultural exchange. Biodiversitas 2000; 31: 11-14.

13. National System of Phytogenetic Resources for Food and Agriculture (SINAFERI). Generalities of the quelites. SINAFERI Red quelites., Mexico city, 2015.

14. National Commission for the Knowledge and Biodiversity Use (CONABIO). Mexican weeds: Papalo-quelite, verdolaga, quintonil, romerito, quelite cenizo, huazontle, alaches, epazote, chaya de monte, hoja santa, chipil. CONABIO., Mexico city, 2009.

15. Gálvez-Mariscal A, Peña-Montes C. Revaluation of the traditional Mexican diet: an interdisciplinary vision. RDU 2015; 16(5): 2-15.

16. Jiménez-Aguilar D, Grusak M. Minerals, vitamin C, phenolics, flavonoids and antioxidant activity of Amaranthus leafy vegetables. J Food Compost Anal 2017; 58: 33-39.

17. Linares E, Balcázar T, Bye R. The Mexican wild vegetables, the quelites; former comrades today almost forgotten. Botanic Gardens Conservation International., Mexico city, 2006.

18 Solis-Becerra C, Estrada-Lugo E. Culinary practices and recognition of the local diversity of wild vegetables in the collective women and corn of Teopisca, Chiapas, Mexico. LiminarR 2014; 12(2): 148-162.

19. López-García G, Baeza-Jiménez R, Garcia-Galindo H, DublánGarcía O, Lopez-Martinez L. Cooking treatments effect on bioactive compounds and antioxidant activity of quintonil (Amaranthus hybridus) harvested in spring and fall seasons. CyTA-J Food 2018; 16(1): 707-714.

20. Slabbert M, Krüger G. Antioxidant enzyme activity, proline accumulation, leaf area and cell membrane stability in water stressed Amaranthus leaves. S African J Bot 2014; 95: 123-128.

21. Aziz A, Akram N, Ashraf M. Influence of natural and synthetic vitamin $C$ (ascorbic acid) on primary and secondary metabolites and associated metabolism in quinoa (Chenopodium quinoa Willd.) plants under water deficit regimes. Plant Physiol Biochem 2018; 123: 192-203.

22. Jin R, Wang Y, Liu R, Gou J, Chan Z. Physiological and Metabolic Changes of Purslane (Portulaca oleracea L.) in Response to Drought, Heat, and Combined Stresses. Front Plant Sci 2016; 6: 1123.

23. López-Mejía A, López-Malo A, Palou E. Antioxidant capacity of extracts from amaranth (Amaranthus hypochondriacus L.) 
seeds or leaves. Ind Crops Prod 2014; 53: 55-59.

24. Bhargava A, Shukla S, Srivastava J, Singh N, Ohri D. Genetic diversity for mineral accumulation in the foliage of Chenopodium spp. Sci Hortic 2008; 118(4): 338-346.

25. Hsu B, Lin S, Inbaraj B, Chen B. Simultaneous determination of phenolic acids and flavonoids in Chenopodium formosanum Koidz. (djulis) by HPLC-DAD-ESI-MS/MS. J Pharm Biomed Anal 2016; 132: 109-116.

26. Nowak R, Szewczyk K, Gawlik-Dziki U, Rzymowska J, Komsta L. Antioxidative and cytotoxic potential of some Chenopodium L. species growing in Poland. Saudi J Biol Sci 2016; 23(1): 15-23.

27. Bhargava A, Shukla S, Dixit B, Bannerji R, Ohri D. Variability and genotype cutting interactions for different nutritional components in Chenopodium album L. Hort Sci 2006; 33(1): 29-38.

28. Bhargava A, Shukla S, Ohri D. Evaluation of foliage yield and leaf quality traits in Chenopodium spp. in multiyear trials. Euphytica 2007; 153: 99-213.

29. Erkan N. Antioxidant activity and phenolic compounds of fractions from Portulaca oleracea L. Food Chem 2012; 133(3): 775-781.

30. Alam M, Juraimi A, Rafii M, Abdul Hamid A, Aslani F, Hasan M, Mohd Zainudin M, Uddin M. Evaluation of antioxidant compounds, antioxidant activities, and mineral composition of 13 collected purslane (Portulaca oleracea L.) accessions. Biomed Res Int 2014; Biomed Res Int 2014. http://dx.doi. org/10.1155/2014/296063

31 Swarna J, Lokeswari TS, Smita M, Ravindhran R. Characterization and determination of in vitro antioxidant potential of betalains from Talinum triangulare (Jacq.) Willd. Food Chem 2013; 141(4): 4382-4390.

32. Miyazaki Y, Ichimuraa A, Sato S, Fujii T, Oishi S, Sakai H, Takeshima $H$. The natural flavonoid myricetin inhibits gastric H+, K+ -APase. Eur J Pharmacol 2018; 820: 217-221.

33. Li H, Deng Z, Liu R, Zhu H, Draves J, Marcone M, Sun Y, Tsao $R$. Characterization of phenolics, betacyanins and antioxidant activities of the seed, leaf, sprout, flower and stalk extracts of three Amaranthus species. I Food Compost Anal 2015; 37: 75-81.

34. Kokanova-Nedialkova Z, Bücherl D, Nikolov S, Heilmann I, Nedialkov P. Flavonol glycosides from Chenopodium foliosum Asch. Phytochem Lett 2011; 4(3): 367-371.

35. Fasuyi A. Bio-nutritional evaluations of three tropical leaf vegetables (Telfairia occidentalis, Amaranthus cruentus and Talinum triangulare) as sole dietary protein sources in rat assay. Food Chem 2007; 103(3): 757-765.

36. Simopoulos A. Omega-3 fatty acids and antioxidants in edible wild plants. Eur J Biol Res 2004; 37(2): 263-277.

37. Girija K, Lakshman K. Anti-hyperlipidemic activity of methanol extracts of three plants of Amaranthus in triton-WR 1339 induced hyperlipidemic rats. Asian Pac J Trop Biomed 2011; 1(1): S62-S65.

38. Kumar B, Lakhman S, Jayaveera K, Shekar D, Nandeesh R, Velmurugan $C$. Chemoprotective and antioxidant activities of methanolic extract of Amaranthus spinosus leaves on paracetamol induced liver damage in rats. Acta Med Sal 2010; 39(2): 68-74.

39. Kumar B, Lakhman K, Jayaveera $K$. In vitro anthelmintic property of methanol extract of Amaranthus viridis. EJEAFChe 2010; 9: 1093-1097.

40. Ibrahim M, Pieters $R$, Van der Walt A, Bezuidenhout C, AbdelAzeim S, Abdel-wahhab M. Protective effect of traditional
African vegetable (Amaranthus hybridus) against aflatoxin B1 and/or fumonisin B1 in a rat hepatoma cell-line. Toxicol Lett 2013; 221S: S59-S256.

41. Kumar B, Lakhman K. Hepatoprotective activity of methanol extract of Amaranthus caudatus against paracetamol induced hepatic injury in rats. Chin J Integr Med 2011; 9(2): 194-200.

42. Sharma A, Flores-Vallejo R, Cardoso-Taketa A, Villarreal M. Antibacterial activities of medicinal plants used in Mexican traditional medicine. J Ethnopharmacol 2016; 208: 264-329.

43. Gawlik-Dziki U, Swieca M, Sułkowski M, Dziki D, Baraniak B, Czyz J. Antioxidant and anticancer activities of Chenopodium quinoa leaves extracts - In vitro study. Food Chem Toxicol 2013; 57: 154-160.

44. Kokanova-Nedialkova $Z$, Nedialkov $P$, Kondeva-Burdina $M$, Simeonova $R$, Tzankova $V$, Aluani D. Chenopodium bonus-henricus L.- A source of hepatoprotective flavonoids. Fitoterapia 2017; 118: 13-20.

45. Shen H, Tang G, Zeng G, Yang Y, Cai X, Li D, Liu H, Zhou N. Purification and characterization of an antitumor polysaccharide from Portulaca oleracea L Carbohydr Polym 2013; 93(2): 395-400.

46. Oliveira J, Costa A, Lima David J, David J, Giulietti A, Paganucci L, Ribeiro R, Pereira Soares M. Anti-leishmanial and immunomodulatory activities of extracts from Portulaca hirsutissima and Portulaca werdermannii. Fitoterapia 2007; 78(7-8): 510-514.

47. Wanyin W, Liwei D, Lin J, Hailiang X, Changquan L, Min L. Ethanol extract of Portulaca oleracea L. protects against hypoxia-induced neurodamage through modulating endogenous erythropoietin expression. J Nutr Biochem 2012; 23(4): 385391.

48. Chang-Quan W, Gui-Qin Y. Betacyanins from Portulaca oleracea $L$. ameliorate cognition deficits and attenuate oxidative damage induced by D-galactose in the brains of senescent mice. Phytomedicine 2010; 17(7): 527-532.

49. Al-Quraishy S, Dkhil M, Abdel-Moneim A. Protective effects of Portulaca oleracea against rotenone mediated depletion of glutathione in the striatum of rats as an animal model of Parkinson's disease. Pestic Biochem Physiol 2012; 103(2): 108-114.

50. Shukla S, Bhargava A, Chatterjee A, Srivastava J, Singh N, Singh S. Mineral profile and variability in vegetable Amaranth (Amaranthus tricolor). Plant Foods Hum Nutr 2006; 61(1): 23-28

51. Ngugi C, Oyoo-Okoth E, Manyala J, Fitzsimmons K, Kimotho A. Characterization of the nutritional quality of amaranth leaf protein concentrates and suitability of fish meal replacement in Nile tilapia feeds. Aquaculture Reports 2017; 5: 62-69.

52. Alvarez-Jubete $L$, Wijngaard $H$, Arendt $E$, Gallagher $E$. Polyphenol composition and in vitro antioxidant activity of amaranth, quinoa buckwheat and wheat as affected by sprouting and baking. Food Chem 2010; 119(2): 770-778.

53. Barba de la Rosa A, Fomsgaard I, Laursen B, Mortensen A, Olvera-Martínez L, Silva-Sánchez C, Mendoza-Herrera A, González-Castañeda J, De León-Rodríguez A. Amaranth (Amaranthus hypochondriacus) as an alternative crop for sustainable food production: phenolic acids and flavonoids with potential impact on its nutraceutical quality. I Cereal Sci 2011; 49(1): 117-121.

54. Arora S, Itankar P, Verma $P$, Bharne A, Kokare D. Involvement of $N F \kappa B$ in the antirheumatic potential of Chenopodium album L., aerial parts extracts. J Ethnopharmacol 2014; 155(1): 222-229. 
55. Yan J, Li-Rong S, Zhong-Yu Z, Yu-Chan C, Wei-Min Z, Hao-Fu $D$, Jian-Wen T. Homoisoflavonoids from the medicinal plant Portulaca oleracea. Phytochemistry 2012; 80: 37-41.

56. Xiang L, Xing D, Wang W, Wang R, Ding Y, Du L. Alkaloids from Portulaca oleracea L. Phytochemistry 2005; 66(21): 2595-2601.

57. Liang X, Tian J, Li L, Gao J, Zhang Q, Gao P, Song, S. Rapid determination of eight bioactive alkaloids in Portulaca oleracea $L$. by the optimal microwave extraction combined with positive-negative conversion multiple reaction monitor (p/ MRM) technology. Talanta 2014; 120: 167-172.

58. Hai-Liang X, Yin-Huan H, Yan-Feng X, Xiao-Qiang Y, Min L, Jin-Cai L, Chang-Quan. Portulacerebroside A: New Cerebroside from Portulaca oleracea L. Chin I Nat Med 2008; 6(6): 401403.

59. Kumar B, Lakhman K, Jayaveera K. Antinociceptive and antipyretic activities of methanol extract Amaranthus caudatus. Lat Am J Pharm 2010; 29(4): 635-639.

60. Lakshman K. Hepatoprotective and antioxidant activities of Amaranthus viridis. Maced I Med Sci 2011; 4(2): 1-6.

61. Pandhare $R$, Balakrishnan S, Mohite $P$, Khanage S. Antidiabetic and anti-hyperlipidaemic potential of Amaranthus viridis (L.) Merr. in streptozotocin induced diabetic rats. Asian Pac J Trop Dis 2012; 2: S180-S185.

62. Kaur N, Dhuna V, Kamboja S, Agrewala J, Singh J. A novel antiproliferative and antifungal lactin from Amaranthus viridis Linn. seeds. Protein Peptide Lett 2006; 13(9): 897-905.

63. Obi R, Iroagba I, Ojiako O. Virucidal potential of some edible Nigerian vegetables. Afr J Biotechnol 2006; 5(19): 1785-1788.
64. Salvamani S, Gunasekaran B, Shukor M, Shaharuddin N, Sabullah M, Ahmad S. Anti-HMG-CoA reductase, antioxidant, and anti-inflammatory activities of Amaranthus viridis leaf extract as a potential treatment for hypercholesterolemia. Evid Based Complement Alternat Med 2016; 11: 1-10.

65. Limaverde $P$, Campina $F$, da Cunha F, Crispim F, Figueredo $F$, Lima L, Oliveira-Tintino C, Balbino V. Inhibition of the TetK efflux-pump by the essential oil of Chenopodium ambrosioides L. and $\alpha$-terpinene against Staphylococcus aureus IS-58. Food Chem Toxicol 2017; 109(Pt 2): 957-961.

66. Guarrera P, Savo V. Perceived health properties of wild and cultivated food plants in local and popular traditions of Italy: A review. J Ethnopharmacol 2013; 146(3): 659-680.

67. Sikarwar I, Dey Y, Wanjari M, Sharma A, Gaidhani S, Jadhav A. Chenopodium album Linn. leaves prevent ethylene glycolinduced urolithiasis in rats. J Ethnopharmacol 2016; 195: 275-282.

68. Yadav N, Vasudeva N, Singh S, Sharma S. Medicinal properties of genus Chenopodium Linn. Nat prod Radiance 2007; 6(2): 131-134.

69. Monzote L, Pastor J, Scull R, Gille L. Antileishmanial activity of essential oil from Chenopodium ambrosioides and its main components against experimental cutaneous leishmaniasis in BALB/C mice. Phytomedicine 2014; 21(8-9): 1048-1052.

70. Gu JF, Zheng Z, Yuan J, Zhao B, Wang C, Zhang L, Xu Q, Jia $X$. Comparison on hypoglycemic and antioxidant activities of the fresh and dried Portulaca oleracea L. in insulin-resistant HepG2 cells and streptozotocin-induced C57BL/6J diabetic mice. J Ethnopharmacol 2014; 161: 214-223. 